\title{
Nota sobre algunas plantas de Marruecos
}

\author{
Ana Ortega-Olivencia ${ }^{1} \&$ Juan Antonio Devesa ${ }^{2}$ \\ ${ }^{1}$ Área de Botánica, Facultad de Ciencias, Universidad de Extremadura, Avenida de Elvas, s.n., 06006 Badajoz, España. \\ 2Departamento de Botánica, Ecología y Fisiología Vegetal, Facultad de Ciencias, Universidad de Córdoba, Campus de \\ Rabanales, Edificio José Celestino Mutis, Ctra. de Madrid km. 396 A, 14014 Córdoba, España.
}

\section{Correspondencia}

Ana Ortega Olivencia

e-mail: aortega@unex.es

Recibido: 12 marzo 2021

Aceptado: 8 abril 2021

Publicado on-line: 7 mayo 2021

Editado por: Marta Recio Criado

\section{Resumen}

Tras la identificación de numerosas plantas recolectadas en una expedición a Marruecos durante 1989, nuestro objetivo fue averiguar si alguna implicaba la ampliación o confirmación de área para algunos de los sectores considerados en la Checklist of Vascular Plants of $N$ Morocco. Con los resultados obtenidos se amplía la información corológica de tres taxones de angiospermas, recolectados en los sectores de Targuist (v.g. Sedum andegavense), Tsoul (v.g. Jasione montana subsp. cornuta) y Zerhoun (v.g. Mantisalca duriaei). Solo un taxón es endémico de Marruecos (Jasione montana subsp. cornuta).

Palabras clave: Flora, Marruecos, Rif

\section{Abstract}

Note on some plants from Morocco

After identifying numerous plants collected in an expedition to Morocco during 1989, our aim was to find out if any of them implied the expansion or confirmation of area for some of the sectors considered in the Checklist of Vascular Plants of N Morocco. The results indicate that the area of three angiosperm taxa is expanded, specifically from the Targuist (e.g. Sedum andegavense), Tsoul (e.g. Jasione montana subsp. cornuta) and Zerhoun (e.g. Mantisalca duriaei) sectors. Only one taxon is endemic to Morocco (Jasione montana subsp. cornuta).

Keywords: Flora, Morocco, Rif
Durante los días 8 a 13 de mayo de 1989, varios profesores de las universidades de Córdoba, Extremadura y Málaga realizaron una expedición por Marruecos recolectando plantas en algunas localidades del Atlas Medio occidental (Azrou, Tiouririne, Ain Leuh, Khenifra, Zeida) y oriental (Djebel Tazzeka), Sáhara marroquí (Midelt, ErRachidía, Erfoud, Merzouga), zonas costeras (Larache, Alhoceima) y el Rif (Targhist, Ketama, Djebel Tidirhine), entre otras. Se recolectaron cerca de 200 pliegos, de los cuales unos 150 se encuentran depositados en el herbario UNEX y de los que mayoritariamente también hay duplicados en los herbarios COFC, MGC y SEV. La identificación de estos materiales ha evidenciado que para tres taxones se amplía su área a algunos sectores cubiertos en la Checklist of Vascular Plants of $N$ Morocco (Valdés et al., 2002; en adelante Checklist), como Targuist, Tsoul y Zerhoun, y uno de ellos se cita por primera vez para la misma. Para la realización de este trabajo se han consultado numerosas publicaciones, las cuales se citan junto con los taxones, y a las que hay que añadir la de Fennane \& lbn Tattou (2005).

A continuación, se indican los taxones por orden alfabético de géneros y especies/subespecies, mostrándose para cada sector la enumeración con la que aparece consignado en la Checklist.

1. Jasione montana subsp. cornuta (Ball) Greuter \& Burdet

Tsoul (13): Tsoul, Matmata, 11.05.1989, bordes de cultivo, B. Cabezudo, J. Devesa, E. Domínguez, J.M. Nieto, E. Ruiz-Clavijo \& R. Tormo (MGC 27832, UNEX 37590-1).

Taxón endémico de Marruecos (El Oualidi et al., 2012; Rankou et al., 2013), que Fennane \& Mathez (2014) no reconocen e incluyen entre las sinonimias de Jasione montana. Peris et al. (2002) solo indican su presencia en el sector de Tánger, aunque posteriormente varios autores (Mateos \& Valdés, 2003, 2010; Valdés et al., 2005; Chambouleyron, 2012) amplían su área a varios sectores más, como el Rif occidental, Ouezanne y la península tingitana. 
Se extiende con esta cita su área al sector de Tsoul.

\section{Mantisalca duriaei (Spach) Briq. \& Cavill.}

Zerhoun (9): Zerhoun-Saiss Nord, entre Fès y BirTam-Tam, 11.05.1989, cultivos, B. Cabezudo, J. Devesa, E. Domínguez, J.M. Nieto, E. Ruiz-Clavijo \& R. Tormo (MGC 28295, UNEX 37717-1).

Especie endémica del $W$ de la región Mediterránea (Ruiz de Clavijo \& Devesa, 2014) que no es indicada para ningún sector cubierto por la Checklist (Susanna \& Garcia Jacas, 2002), pues solo se menciona para el territorio M. salmantica (L.) Briq. \& Cavill. No obstante, Fennane (2014) la señala para el Atlántico medio marroquí (entre Casablanca y Benslimane), el Atlántico norte (Taza; Fès; Khnichète; Tissane), las mesetas del Marruecos oriental (Taourirt), el litoral mediterráneo (con interrogación) y el Rif, señalando que se necesita precisar su distribución. Es una especie catalogada como rara por Fennane \& Ibn Tattou (1998).

\section{Sedum andegavense (DC.) Desv.}

Targuist (8): Targuist-Jebha, Ketama, subida al Djebel Tidirhine, 12.05.1989, cultivos de Cannabis y pinar, B. Cabezudo, J. Devesa, E. Domínguez, J.M. Nieto, E. Ruiz-Clavijo \& R. Tormo (MGC 27840, UNEX 37597-1).

Esta especie no ha sido indicada en la Checklist (Springate, 2002), ni tampoco la citan El Alaoui Faris \& Ibn Tattou (1999) para la zona rifeña, ya que solo precisan su existencia en el Anti-Atlas, Alto Atlas y Atlas Medio. A nivel general, se distribuye por el SW de Europa y el W de la región Mediterránea (Castroviejo \& Velayos, 1997).

\section{Agradecimientos}

Nuestro agradecimiento al Dr. José García Sánchez, conservador del herbario MGC (Universidad de Málaga), por proporcionarnos información requerida para muchos de los pliegos recolectados en la expedición; igualmente, al personal encargado de los herbarios COFC y UNEX y a dos evaluadores anónimos por sus comentarios.

\section{Referencias}

Castroviejo, S. \& Velayos, M. (1997) Sedum L. In S. Castroviejo, C. Aedo, M. Laínz, R. Morales, F. Muñoz Garmendia, G. Nieto Feliner \& J. Paiva (Eds.), Flora iberica. Vol. V: 121-153. Madrid: Real Jardín Botánico, CSIC.

Chambouleyron, M. (2012) Contribution a la connaissance de la flore de la péninsule tingitane (Maroc). Lagascalia, 32, 35-227.

El Alaoui Faris, F.Z. \& Ibn Tattou, M. (1999) Sedum L. In M. Fennane, M. Ibn Tattou, J. Mathez, A. Ouyahya \& J. El Oualidi (Eds.), Flore pratique du Maroc. Manuel de détermination des plantes vasculaires 1: 466-474. Rabat: Travaux de I'Institut Scientifique, Série Botanique, № 36 .
El Oualidi, J., Khamar, H., Fennane, M., Ibn Tattou, M., Chauvet, S. \& Taleb, M.S. (2012) Checklist des endémiques et spécimens types de la flore vasculaire de l'Afrique du Nord. Documents de L'Institut Scientifique, 25, 1-189.

Fennane, M. (2014) Mantisalca Cass. In M. Fennane, M. Ibn Tattou \& J. El Oualidi (Eds.), Flore pratique du Maroc. Manuel de détermination des plantes vasculaires 3: 154-155. Rabat: Travaux de l'Institut Scientifique, Série Botanique, $n^{\circ} 40$.

Fennane, M. \& Ibn Tattou, M. (1998) Catalogue des plantes vasculaires rares, menacées ou endémiques du Maroc. Bocconea, 8, 5-243.

Fennane, M. \& Ibn Tattou, M. (2005) Flore vasculaire du Maroc: Inventaire et chorologie. Vol. 1. Rabat: Travaux de I'Institut Scientifique, Sér. Botanique, $n^{\circ} 37$.

Fennane, M. \& Mathez, J. (2014) Jasione L. In M. Fennane, M. Ibn Tattou \& J. El Oualidi (Eds.), Flore pratique du Maroc. Manuel de détermination des plantes vasculaires 3: 73-75. Rabat: Travaux de l'Institut Scientifique, Série Botanique, $n^{\circ} 40$.

Mateos, M.A. \& Valdés, B. (2003) Nuevos taxones para el Rif occidental. II. Acta Botanica Malacitana, 23, 265-273.

https://doi.org/10.24310/abm.v28i0.7302

Mateos, M.A. \& Valdés, B. (2010) Catálogo de la flora vascular del Rif occidental calizo ( $\mathrm{N}$ de Marruecos). II. Caesalpiniaceae-Compositae. Lagascalia, 30, 47-303.

Peris, J.B., Romo, A. \& Stübing, G. (2002) Jasione L. In B. Valdés, M. Rejdali, A. Achhal, S.L. Jury \& J.M. Montserrat (Eds.), Checklist of vascular plants of N Morocco with identification keys. Vol. 2: 597-598. Madrid: CSIC.

Rankou, H., Culham, A., Jury, S.L. \& Christenhusz, M.J.M. (2013) The endemic flora of Morocco. Phytotaxa, 78, 1-69.

http://dx.doi.org/10.11646/phytotaxa.78.1.1

Ruiz de Clavijo, E. \& Devesa, J.A. (2014) Mantisalca Cass. In J.A. Devesa, A. Quintanar \& M.Á. García (Eds.), Flora lberica. Vol. XVI(1): 290-298. Madrid: Real Jardín Botánico, CSIC.

Springate, L.S. (2002) Sedum L. In B. Valdés, M. Rejdali, A. Achhal, S.L. Jury \& J.M. Montserrat (Eds.), Checklist of vascular plants of N Morocco with identification keys. Vol. 1: 276-280. Madrid: CSIC.

Susanna, A. \& Garcia Jacas, N. (2002) Mantisalca Cass. in Cuvier. In B. Valdés, M. Rejdali, A. Achhal, S.L. Jury \& J.M. Montserrat (Eds.), Checklist of vascular plants of $N$ Morocco with identification keys. Vol. 2: 717. Madrid: CSIC.

Valdés, B., Rejdali, M., Achhal, A., Jury, S.L. \& Montserrat, J.M. (2002) (Eds.), Checklist of vascular plants of $N$ Morocco with identification keys. Madrid: CSIC.

Valdés, B., Santa-Bárbara, C. \& Pina, F.J. (2005) Novedades corológicas para la comarca de Ouezzane ( $\mathrm{N}$ de Marruecos). Lagascalia, 25, 234240. 